\title{
Coupling, Scattering, and Perturbation Theory: Semi-analytical Analyses of Photonic-Crystal Waveguides
}

\author{
Steven G. Johnson, M. L. Povinelli, P. Bienstman, M. Skorobogatiy,* M. Soljačić, \\ M. Ibanescu, E. Lidorikis, and J. D. Joannopoulos \\ Dept.of Physics, Massachusetts Institute of Technology, stevenj@alum.mit.edu \\ * OmniGuide Communications, Inc., Cambridge MA 02139
}

\begin{abstract}
Although brute-force simulations of Maxwell's equations, such as FDTD methods, have enjoyed wide success in modeling photonic-crystal systems, they are not ideally suited for the study of weak perturbations, such as surface roughness or gradual waveguide transitions, where a high resolution and/or large computational cells are required. Instead, we suggest that these important problems are ideally suited for semi-analytical methods, which employ perturbative corrections (typically only needing the lowest order) to the exactly understood perfect waveguide. However, semi-analytical methods developed for the study of conventional waveguides require modification for high index-contrast, strongly periodic photonic crystals, and we have developed corrected forms of coupled-wave theory, perturbation theory, and the volume-current method for this situation. In this paper, we survey these new developments and describe the most significant results for adiabatic waveguide transitions and disorder losses. We present design rules and scaling laws for adiabatic transitions. In the case of disorder, we show both analytically and numerically that photonic crystals can suppress radiation loss without any corresponding increase in reflection, compared to a conventional strip waveguide with the same modal area, group velocity, and disorder strength.
\end{abstract}

\section{INTRODUCTION}

Photonic crystals, periodic dielectric structures with a band gap that prohibits the propagation of light in a range of wavelengths, offer tantalizing new possibilities for controlling and designing optical phenomena [1]. In order to employ them in practical devices, especially in potential high-density integrated optical systems, however, one must gain a greater understanding of scattering loss mechanisms in such crystal structures. This task that is made more challenging by the use of high index contrasts, tight confinement, and strong periodic modulation, which invalidate many semi-analytical tools that were previously employed in more conventional optical waveguides. One approach that has been successful in many cases to employ brute-force simulation, such as FDTD methods, which model the full Maxwell's equations with approximations only in the finite resolution and in the boundary conditions. Brute force simulation becomes more challenging and less illuminating, however, when applied to problems such as disorder-induced scattering and losses from slow transitions, due to the high spatial resolution and weak effects that those phenomena embody, as well as the large variety of scatterers that one might like to consider. It is these situations that we study in this paper, and we exploit their key property of involving small perturbations to an ideal system in order to develop efficient semi-analytical tools and even general analytical predictions. We are able to show, for example, that an adiabatic theorem applies to slow transitions in photonic crystals and periodic waveguides (and what scaling laws the losses follow as the length and group velocity of the waveguide are changed). For roughness/disorder losses, we are able to prove that, compared to an equivalent conventional waveguide (equal mode size, group velocity, and disorder strength), radiative scattering is suppressed and reflections are no worse in a photonic-crystal waveguide (rather than being increased as radiation is suppressed as one might fear).

For a perturbation $\Delta \varepsilon(\mathbf{x})$ in the dielectric function $\varepsilon(\mathbf{x})$, a key quantity is the volume current $\mathbf{J} \sim \Delta \varepsilon \cdot \mathbf{E}$, where $\mathbf{E}$ is an electric field of the unperturbed system (whose solution is already known). This current is central to the computation of low-order corrections to electromagnetic eigenstates and eigenvalues in perturbation theory, is used for the coupling matrix element in coupled-wave theory, and acts as an oscillating source term for a Green's function formalism. As we shall discuss below, all three of these are promising approaches in the analytical and semi-analytical study of small perturbations and slow waveguide transitions. First, however, we address an important problem in high index-contrast systems, that of the proper evaluation of $\Delta \varepsilon \cdot \mathbf{E}$ when $\Delta \varepsilon$ comes from a perturbed boundary between two dielectrics - in this case, the $\Delta \varepsilon \cdot \mathbf{E}$ current must be modified to avoid problems (incorrect, or even ill-defined expressions) due to the discontinuity in the electric field at dielectric interfaces. 
Overall, we attempt to highlight the main results of these techniques and their implications for the study of losses in photonic crystals, referring to other papers for detailed derivations and extensive numerical results.

\section{CORRECT TREATMENT OF BOUNDARY PERTURBATIONS}

Consider a case where the boundary between two dielectrics, $\varepsilon_{1}$ and $\varepsilon_{2}$, is shifted towards $\varepsilon_{2}$ by some distance $h$, and in particular the case where $h \rightarrow \Delta h$ is small (e.g. from disorder) so as to serve as a perturbative expansion parameter. The relevant quantity that appears in perturbation theory, coupled-wave theory, and Green's functions is a volume integral of the form $\int \mathbf{F} \cdot \mathbf{J}$ where $\mathbf{F}$ is an electric field of the unperturbed system or a Green's function (tensor) and $\mathbf{J}$ is nominally $\sim \Delta \varepsilon \cdot \mathbf{E}$. For the case of a small boundary perturbation, this becomes the surface integral $\int \mathbf{F} \cdot \mathbf{E} \cdot \Delta \varepsilon_{12} \Delta h$, where $\Delta \varepsilon_{12} \equiv \varepsilon_{1}-\varepsilon_{2}$. Such a surface integral, however, is problematic because $\mathbf{E}$ (or at least its perpendicular component) is discontinuous across a dielectric interface; on which side does one evaluate the field?

In [2], we showed that in fact this naïve surface integral leads to incorrect results in perturbation theory, regardless of which side the electric field is evaluated on. A similar result holds for coupled-wave theory (which is closely related to perturbation theory), as well as for Green's functions: intuitively, the basic requirement in order to obtain mathematically well-defined expressions turns out to be that $\int \mathbf{J} \cdot \mathbf{E}$, the power scattered by the perturbation, have the same magnitude no matter which side of the interface one considers. In order to obtain the correct expression, one can consider the discontinuous structure as the limit of smoothed structures where $\varepsilon(\mathbf{x})$ changes continuously. A well-defined (and thus unique) limit is found by performing this smoothing anisotropically as suggested by effective-medium theory, and the result to lowest order in $\Delta h$ is that [2]:

$$
\mathbf{J} \sim \Delta \varepsilon \cdot \mathbf{E} \rightarrow \delta\left(\mathbf{x}_{\perp}-x_{\text {surf }}\right)\left[\Delta \varepsilon_{12} \mathbf{E}_{\|}-\varepsilon \Delta\left(\varepsilon_{12}^{-1}\right) \mathbf{D}_{\perp}\right] \Delta h
$$

where the Dirac delta function $\delta(\cdots)$ is to obtain a surface integral, $\Delta\left(\varepsilon_{12}^{-1}\right) \equiv \varepsilon_{1}^{-1}-\varepsilon_{2}^{-1}, \mathbf{E}_{\|}$is the component of $\mathbf{E}$ parallel to the interface, and $\mathbf{D}_{\perp}$ is the component of $\mathbf{D}=\varepsilon \mathbf{E}$ perpendicular to the interface. In this way, $\mathbf{J} \cdot \mathbf{E}$ only has terms proportional to $\left|\mathbf{E}_{\|}\right|^{2}$ and $\left|D_{\perp}\right|^{2}$, both of which are continuous across the interface.

\section{COUPLED-WAVE THEORY FOR PERIODIC WAVEGUIDES}

Conventional dielectric and metallic waveguides are uniform along the propagation direction $(z)$, but that is not really a requirement: thanks to the Bloch-Floquet theorem, light can propagate without scattering down a waveguide as long as it is periodic with some period (pitch) $\Lambda$. This fact has been exploited to design intrinsically lossless waveguides by, for example, linear defects introduced in photonic crystals with complete band gaps [1] as well as in hybrid systems (photonic-crystal slabs) that combine bandgap-guiding with index-guiding [3]. However, the strong periodic modulation (often 3:1 or greater index contrast) of even the perfect photonic crystal waveguide requires substantial revision of past semi-analytical methods that were used to study waveguide imperfections. In particular, we present a new generalization of classical coupled-wave theory that is adapted for periodic systems.

Coupled-wave (or coupled-mode) theory treats a $z$-varying/imperfect waveguide by expressing the fields $|\psi\rangle$ at each $z$ as an expansion in the eigenmodes $|n\rangle$ of a perfect (uniform) waveguide with some expansion coefficients $c_{n}(z)$ [4-6]. (Here, we employ the Dirac notation of an abstract Hilbert space of states $|\psi\rangle$ with inner products $\langle\phi \mid \psi\rangle$ and linear operators $\hat{O}$.) One then finds the coupled-wave equations for $c_{n}$, of the form $d c_{n} / d z=\sum_{m} M_{n m} c_{m} e^{i \int \Delta \beta d z^{\prime}}$ for some coupling matrix $M$ and phase mismatches $\Delta \beta_{n m}$. The eigenmodes of uniform waveguides, however, form a poor basis for strongly-periodic structures, because they yield rapidly varying $c_{n}$ 's even for the case of a perfect (periodic) waveguide where one knows analytically that there is no scattering. Instead, we have for the first time developed exact, continuous coupled-wave equations as a function of $z$ (in contrast to previous work as a function of time [7]) by expanding in the Bloch eigenmodes of the periodic system $[8,9]$.

In order to derive coupled-wave equations from the overall algebraic structure of electromagnetism without the usual muddle of curls and cross products, we also developed a quantum-mechanics-like abstract operator formalism $\hat{A}|\psi\rangle=-i \hat{B} \frac{\partial}{\partial z}|\psi\rangle$ for the $z$-evolution of the state $|\psi\rangle$ (expressed via the four transverse field components) in terms of Hermitian linear operators $\hat{A}$ and $\hat{B}$ encapsulating the exact Maxwell's equations at a constant $\omega[10,11]$. In this way, the Bloch eigenmodes $e^{i \beta_{n} z}|n\rangle$ (where $|n\rangle$ is periodic) satisfy a generalized Hermitian eigenproblem 
for the Bloch wavenumber $\beta$ (as distinct from the more-typical eigenproblem with $\omega^{2}$ eigenvalues [1]); properties such as orthogonality then follow automatically from the usual theorems of linear algebra.

\subsection{Coupled-wave equations in instantaneous eigenstates}

One classical expansion basis is that of the eigenmodes of a conceptual "instantaneous" uniform waveguide matching the cross section at $z$. In this basis, the scattering $d c_{n} / d z$ depends only on the rate of change of the waveguide cross-section, and one obtains an adiabatic theorem that there is no scattering in the limit of a gradual enough change [12]. Thus, such a basis is well suited for the study of slow taper transitions, e.g. for input/output couplers. A similar basis can be employed for slowly-changing periodic waveguides, by choosing a conceptual periodic waveguide $\varepsilon_{z}(x, y, \tilde{z}+\Lambda)=\varepsilon_{z}(x, y, \tilde{z})$ extending infinitely in a conceptual $\tilde{z}$ space that matches the waveguide cross-section at $z: \varepsilon_{z}(x, y, z)=\varepsilon(x, y, z)$. Such a conceptual waveguide, being constrained only on a single cross section but defined by a periodic unit cell, is not unique, and corresponds to a choice of basis, allowing one to select a convenient slowly-varying family of periodic waveguides [8].

Although the derivation involves a number of algebraic tricks, especially for the case where the period $\Lambda$ is slowly varying (which we do not consider here, for simplicity), the end result is simple. One expands the state $|\psi\rangle$ as a sum $\sum_{n} c_{n}(z)|n\rangle_{z} e^{i \int \beta_{n} d z^{\prime}}$ in the instantaneous Bloch eigenstates $|n\rangle_{z}$ of $\varepsilon_{z}$, and obtains standard-looking ${ }^{1}$ coupled-wave equations $d c_{n} / d z=\sum_{m} M_{n m} c_{m} e^{i \int \Delta \beta d z^{\prime}}$, with coupling coefficients $M_{n m}$ given by [8]:

$$
M_{n m}(z)=\frac{\omega}{c} \int \frac{\partial \varepsilon_{z}}{d z} \mathbf{E}_{n}^{*} \cdot \mathbf{E}_{m}
$$

in terms of the electric fields of the instantaneous Bloch modes at $z$ and the rate of change of the conceptual dielectric function, where the integral is over the unit cell of the (conceptual) periodicity. If the changing dielectric function involves a shifting dielectric boundary, it is critical to use the corrected current $\mathbf{J}$ from Eq. (1) for the $\partial \varepsilon \mathbf{E}$ (where the approximation of small $\Delta h$ is now exact because $d z$ is infinitesimal).

\subsubsection{Consequences for adiabatic transitions}

The knowledge of these rigorous coupled-wave equations has several important consequences for the study of slow waveguide transitions. First of all, because the coupled-wave equations have the standard form, one immediately obtains an adiabatic theorem: as a waveguide changes more and more slowly, scattering disappears and the coefficients $c_{n}$ go to constants. This theorem, however, imposes a condition: the input state(s) must be propagating (not in a gap) and guided (not leaky) for all intermediate "instantaneous" waveguides. This requires some care, especially when operating near a band edge where one can easily shift into a band gap, but is an easy constraint to satisfy once one is aware of it, and leads to simple design rules as discussed in Ref. [8].

Second, by integrating the coupled mode equations analytically to first-order in the coupling rate, e.g. in the approximation the coupling coefficients are nearly constant, one can obtain analytical scaling laws that closely describe exact numerical calculations [8]: (i) as one increases the taper length $L$ of a gradual transition, the loss (mainly reflection) falls like $1 / L^{2}$; (ii) as one approaches a band edge of the dispersion relation, where the group velocity $v_{g}$ goes to zero, the reflection loss increases as $1 / v_{g}^{6}$. Thus, coupling to slow-light states is especially challenging, with $L \sim 1 / v_{g}^{3}$. (In both cases, the overall scaling is multiplied by a sinusoidal envelope due to Fabry-Perot effects.)

Third, the coupled-wave equations provide an efficient semi-analytical method to compute coupling losses in slow transitions, which are otherwise challenging to model because of the spatial resolution requirements they impose on a brute-force method. Here, one simply: (i) computes the eigenmodes and coupling coefficients at a set of intermediate conceptual waveguides, a small calculation involving only a single $\Lambda$ unit cell and a modest resolution unrelated to the taper rate; and (ii) integrate the coupled-wave equations using interpolated coupling coefficients and $\beta$ 's. This is rendered even more efficient by the fact that in the limit of slow transitions one need only integrate the equations to first-order in the rate of change, involving only a single integral; furthermore, for photonic crystals the losses are typically dominated by reflections (due to phase matching and low $v_{g}$ near band

\footnotetext{
${ }^{1}$ One modification is that the eigenvalues $\beta_{n}$ are periodic in $2 \pi / \Lambda$, and the coefficients for these equivalent eigenvalues must be summed at the end to yield the physical solution.
} 

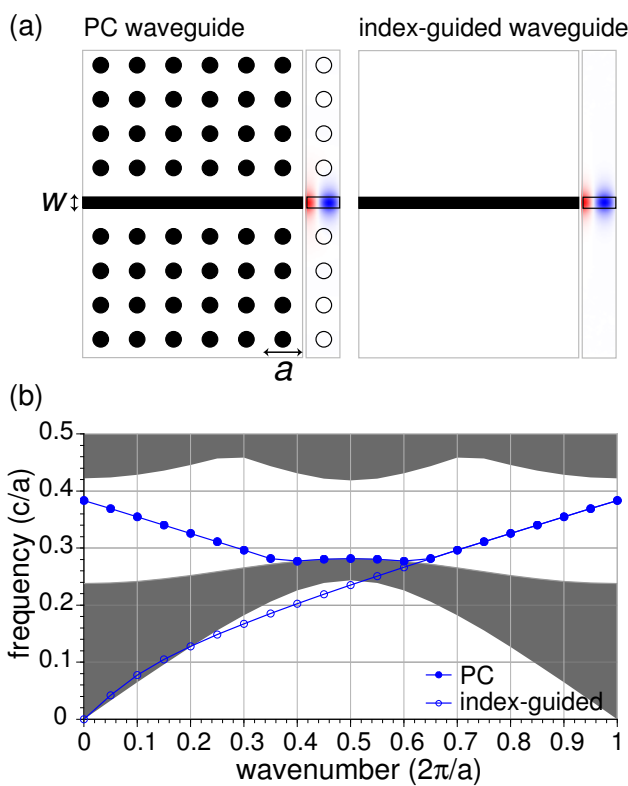

Figure 1: (a) Waveguide geometries and corresponding mode profiles for $\omega=0.31(2 \pi c / a)$. Mode profiles show the electric field component perpendicular to the paper; red and blue indicate negative and positive values respectively. (b) Band diagram for modes of the two waveguides shown in (a). Shaded regions indicate extended TM states of the bulk 2D photonic crystal.

edges), so only two modes need be considered. This method has been demonstrated to yield results accurate within numerical uncertainty compared to a brute-force transfer-matrix technique [8]. Moreover because the coupling coefficients need only be computed once, independent of the taper rate (which acts as an overall scale factor), one can then quickly explore the effect of different taper rates, and even numerically search for an optimal non-uniform taper rate, which might otherwise require thousands of separate transmission calculations. In a couple of hours computation on a personal computer, we have thus been able to find a non-uniform taper rate (tapering more slowly as a band edge is approached) in $2 \mathrm{~d}$ and $3 \mathrm{~d}$ example systems that reduces reflections by two orders of magnitude, compared to a linear taper, over a wide bandwidth within a couple percent of the band edge.

\subsection{Coupled-wave equations in fixed eigenstates}

An instantaneous-eigenstate basis is not as well suited to model small, but not slow, perturbations $\Delta \varepsilon$ (e.g. roughness/disorder) distributed along an otherwise perfectly-periodic waveguide. In this case, it is more convenient to employ the basis of the fixed eigenmodes of the perfect waveguide. Again, the standard coupled-wave theory (in the eigenmodes of uniform waveguides) is not ideal for strongly periodic waveguides, and we have derived a new generalization in terms of the Bloch eigenmodes $|n\rangle$. Using similar algebraic tricks as for the previous section, one obtains the standard form of coupled-wave equations, with coupling matrix elements $M_{n m}$ given by [9]:

$$
M_{n m}(z)=\frac{\omega}{c} \int d^{3} \mathbf{x}^{\prime}\left[\Delta \varepsilon\left(\mathbf{x}_{t}^{\prime}, z\right) \mathbf{E}_{t, n}^{*}\left(\mathbf{x}^{\prime}\right) \cdot \mathbf{E}_{t, m}\left(\mathbf{x}^{\prime}\right)-\Delta \varepsilon^{-1}\left(\mathbf{x}_{t}^{\prime}, z\right) D_{z, n}^{*}\left(\mathbf{x}^{\prime}\right) D_{z, m}\left(\mathbf{x}^{\prime}\right)\right],
$$

in terms of the electric and displacement fields of the $|n\rangle$ and $|m\rangle$ eigenstates, where $t$ denotes the transverse (xy) components . The integration is over the $\Lambda$ unit cell, but note that the perturbation is only evaluated at $z$ to yield the scattering at $z$. (Again, this equation must be modified in a manner similar to Eq. (1) for surface perturbations.) Like for the instantaneous coupled-wave equations, this potentially enables an efficient calculation of reflection and other scattering due to surface roughness, without requiring high resolution or long computational cells. Perhaps more important, however, is what it can tell us analytically about the losses in photonic-crystal waveguides.

\subsubsection{Photonic-crystal vs. conventional waveguide disorder loss}




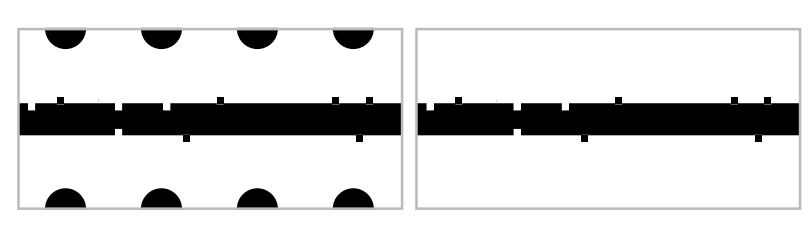

Figure 2: Closeup of central waveguide region, showing how identical surface roughness was added to both the photonic-crystal and index-guided waveguides.

For a photonic crystal waveguide based on a complete band gap, radiative scattering from disorder is impossiblethere are no radiating states to scatter into at the operating frequency in the gap. One might wonder, however, whether this might have an unfortunate side-effect: perhaps some of the disorder-scattered light that would have otherwise radiated is instead reflected? Or, put in another way, a photonic-crystal waveguide is effectively a one-dimensional system (light can only propagate forward or backward), and it is a known theorem of Anderson localization that any amount of disorder in one dimension causes all states to be localizes - this might seem to imply that disorder-induced reflections are worse in one-dimensional systems. If reflection is indeed intrinsically worse in a photonic crystal, this would be a serious problem for optical devices (which are more sensitive to reflection than to loss, because the former produces noise).

Fortunately, Eq. (3) implies that such pessimism is ill-founded. The coupling to a given mode, the reflected mode, only depends upon the (normalized) field amplitude in the disorder, the strength $\Delta \varepsilon$ of the disorder, and the phase mismatch $\Delta \beta$. If these quantities are the same, the reflection will be the same to lowest order (i.e. weak disorder), regardless of what happens to radiative scattering. (Put another way, radiation is suppressed by removing those states entirely, not by redirecting them.)

To illustrate this, we have constructed [9] a simple pair of $2 \mathrm{~d}$ example systems that compares a conventional strip waveguide $(\varepsilon=12$, width $0.3 a$ ) to a photonic crystal waveguide with almost exactly the same field pattern, group velocity, and phase velocity. The latter waveguide is formed by simply embedding the conventional waveguide in a line-defect of a photonic crystal consisting of a $2 \mathrm{~d}$ array of dielectric rods in air (periodicity $a$, radius $0.2 a$ ). The structures, field patterns, and dispersion relations are shown in Fig. 1.The transmission and reflection of these structures was then simulated by an FDTD calculation with resolution of 10 pixels $/ a$, through a disordered region of length $10 a$ created by randomly adding/removing pixels along the edges of the waveguide, as shown in Fig. 2, with probability $p=0.05$. When the resulting transmission/reflections were compared over a $10 \%$ bandwidth, the reflections in the two cases were found to be identical (to within 1\%), with average losses of about 0.07 $\mathrm{dB} / a$ in the strip waveguide and about $0.04 \mathrm{~dB} / a$ in the photonic crystal—over $40 \%$ better, due to the suppressed radiative scattering.

Of course, if one designs a slow-light waveguide with a photonic crystal, losses are worse. Because of the normalization of the states, the coupling matrix elements $M$ are proportional to $1 / \sqrt{v_{g} v_{g}^{\prime}}$, where $v_{g}$ and $v_{g}^{\prime}$ are the group velocities of the incident $(m)$ and scattered $(n)$ modes, respectively. Thus, since weak disorder causes a scattered amplitude $c_{n}$ proportional to $M$, the scattered power $\left|c_{n}\right|^{2}$ is proportional to $1 /\left(v_{g} v_{g}^{\prime}\right)$. So, while scattering into most modes is proportional to $1 / v_{g}$, reflected power is proportional to $1 / v_{g}^{2}$. Matters are improved somewhat if one recalls that for many waveguide devices the length can be reduced proportional to $v_{g}$, in which case the overall reflections are proportional to $1 / v_{g}$. In a few cases, e.g. for enhancing nonlinear effects [13], the length can be reduced proportional to $v_{g}^{2}$, which cancels the loss increase. Still, this is indicative of the challenges in store for the implementation of slow-light devices.

\section{GREEN'S FUNCTION TECHNIQUES}

Finally, let us briefly consider a third semi-analytical technique for studying disorder losses in photonic crystals, one based upon a Green's function formalism. Given a Green's function tensor $\hat{G}_{\omega}\left(\mathbf{x}, \mathbf{x}^{\prime}\right)$ of an perfect system (e.g. a perfectly periodic photonic-crystal waveguide), the scattered field $\mathbf{E}_{s}$ due to a perturbation $\Delta \varepsilon$ from an incident (unperturbed) field $\mathbf{E}_{0}$ at a frequency $\omega$ can be written as:

$$
\mathbf{E}_{s}(\mathbf{x})=\int d^{3} \mathbf{x}^{\prime} \hat{G}_{\omega}\left(\mathbf{x}, \mathbf{x}^{\prime}\right) \Delta \varepsilon\left(\mathbf{x}^{\prime}\right) \mathbf{E}_{0}\left(\mathbf{x}^{\prime}\right)+O\left(\left[\int \Delta \varepsilon \mathbf{E}_{0}\right]^{2}\right)
$$


where we drop the second term for small perturbations, making the first Born approximation. This is also called the volume-current method [6], where the lowest-order scattered field is seen to be the radiation from a localized current source $\mathbf{J} \sim \Delta \varepsilon \mathbf{E}_{0}$. Such a radiation pattern can be computed, for example, by the FDTD method, and the key point is that it does not require a high spatial resolution or an ability to distinguish a small scattered field from the transmitted field. However, in studying a high-index contrast system like a photonic crystal, it is crucial to use the corrected form of the volume current from Eq. (1) for interface perturbations (e.g. roughness), and thus we believe that accurate calculations for photonic crystals with this technique should be possible now for the first time.

Further simplifications are possible. For the common case of completely uncorrelated disorder (surface roughness is only correlated on a few nanometer scale), the radiated power from each pixel of roughness adds incoherently on average, so in this case one can simply compute the radiation from a few individual pixels in a unit cell of the waveguide to obtain the losses. Correlated disorder can also be treated by appropriate Fourier transforms of the radiated fields, and this is a subject that we will treat explicitly in a future publication.

\section{CONCLUSION}

We have surveyed three semi-analytical approaches that we believe promise to answer fundamental questions of losses in photonic crystal waveguides due to small deviations from perfect periodicity. We believe that such techniques offer a compelling alternative to brute force methods such as FDTD for the study of weak imperfections. In all of these approaches, a key component is our development of a corrected effective volume current expression for surface perturbations in high-contrast systems.

Two of these approaches are based on coupled-wave theory, where we have generalized the standard approach to now cover strongly periodic structures. First, in a basis of instantaneous waveguide modes, this yields an efficient description of slow waveguide transitions, where we obtain an adiabatic guarantee of $100 \%$ transmission for sufficiently slow tapers. Moreover, the conditions on the adiabatic theorem provide simple design rules for couplers, and general scaling laws for the strength of the reflection can be derived. We have also shown how such a coupled-wave approach allows efficient computation and optimization of slow tapers that are challenging to model with e.g. FDTD, and have proved accurate in sample-system comparisons. Second, using a basis of the fixed eigenmodes of a perfect system, disorder can be studied, and one has a framework for the direct comparison of photonic-crystal and conventional strip waveguides. There, we have shown that for equivalent modal area, group velocity, and disorder strength, photonic-crystal waveguides can suppress radiation without worsening reflection losses.

Finally, we considered a potential future direction based on the volume-current method, or Green's functions, for computing the scattering losses in photonic crystals. This approach, previously widely used in low index contrast uniform-waveguide systems, has a significant advantage over coupled-wave theory for computing radiative scattering, in that the continuum of radiation eigenmodes need not be solved for explicitly.

\section{Acknowledgments}

This work was supported in part by the Materials Research Science and Engineering Center program of the National Science Foundation under award DMR-9400334.

\section{References}

[1] J. D. Joannopoulos, R. D. Meade, and J. N. Winn, Photonic Crystals: Molding the Flow of Light. Princeton Univ. Press, 1995.

[2] S. G. Johnson, M. Ibanescu, M. A. Skorobogatiy, O. Weisberg, J. D. Joannopoulos, and Y. Fink, "Perturbation theory for maxwell's equations with shifting material boundaries,” Phys. Rev. E, vol. 65, p. 066611, 2002.

[3] S. G. Johnson and J. D. Joannopoulos, Photonic Crystals: The Road from Theory to Practice. Boston: Kluwer, 2002.

[4] D. Marcuse, Theory of Dielectric Optical Waveguides. San Diego: Academic Press, second ed., 1991. 
[5] B. Z. Katsenelenbaum, L. Mercader del Río, M. Pereyaslavets, M. Sorolla Ayza, and M. Thumm, Theory of Nonuniform Waveguides: The Cross-Section Method. London: Inst. of Electrical Engineers, 1998.

[6] A. W. Snyder and J. D. Love, Optical Waveguide Theory. London: Chapman and Hall, 1983.

[7] T. Iizuka and C. M. de Sterke, "Corrections to couipled mode theory for deep gratings," Phys. Rev. E, vol. 61, no. 4, pp. 4491-4499, 2000.

[8] S. G. Johnson, P. Bienstman, M. Skorobogatiy, M. Ibanescu, E. Lidorikis, and J. D. Joannopoulos, "Adiabatic theorem and continuous coupled-mode theory for efficient taper transitions in photonic crystals," Phys. Rev. E, vol. 66, p. 066608, 2002.

[9] M. L. Povinelli, S. G. Johnson, E. Lidorikis, J. D. Joannopoulos, and M. Soljačić, "Reduction of disorderinduced scattering losses using photonic-crystal waveguides." submitted for publication, 2003.

[10] S. G. Johnson, M. Ibanescu, M. Skorobogatiy, O. Weisberg, T. D. Engeness, M. Soljačić, S. A. Jacobs, J. D. Joannopoulos, and Y. Fink, "Low-loss asymptotically single-mode propagation in large-core OmniGuide fibers," Opt. Express, vol. 9, no. 13, pp. 748-779, 2001.

[11] M. Skorobogatiy, S. A. Jacobs, S. G. Johnson, and Y. Fink, "Geometric variations in high index-contrast waveguides, coupled mode theory in curvilinear coordinates," Opt. Express, vol. 10, no. 21, pp. 1227-1243, 2002.

[12] G. Nenciu, "On the adiabatic theorem of quantum mechanics," J. Phys. A, vol. 13, pp. L15-L18, 1980.

[13] M. Soljačić, S. G. Johnson, S. Fan, M. Ibanescu, E. Ippen, and J. D. Joannopoulos, "Photonic-crystal slowlight enhancement of non-linear phase sensitivity," J. Opt. Soc. Am. B, vol. 19, pp. 2052-2059, 2002. 